\title{
A Glimpse of the Body in the Nineteenth Century: A General Paralysis
}

\author{
Soukayna Alami
}

\begin{abstract}
Wallis, Jennifer. Investigating the Body in the Victorian Asylum: Doctors, Patients and Practices. Callaghan, Australia: Palgrave Macmillan, 2017. 276 pages. ISBN 978-3-319-56713-6. Ebook. \$31.00
\end{abstract}

A close look into the body and its parts in patients of asylums has been the focus of pathologists. Cultural historians doing medical humanities like Jennifer Wallis, in turn, take an interest in how pathologists look at the patients and how they interpret their symptoms. Her book Investigating the Body in the Victorian Asylum: Doctors, Patients and Practices, addresses the practices of doctors with respect to the body examination of asylum patients in nineteenth-century asylums, specifically West Riding Asylum in Wakefield, West Yorkshire. The book can be classified as a contribution to medical psychiatry as the writer uses clinical, historical and pathological approaches to explore and report the practices of doctors on asylum patients in the nineteenth century with which they wanted to define the role of the brain in determining the connection between mental illnesses and physical diseases. Wallis's monograph is the result of her long-time engagement with this topic, and as such shows her evolution as an academic. There are several articles of hers leading to, and incorporated in, this monograph, mainly related to the field of anatomy and medical psychiatry since the focus of her research falls on the diseased body of mental patients in nineteenth-century Britain. One of her earlier studies "The Bones of the Insane" (2013), introduces the concept of "fracture death," which occurs in many patients due to falls that happen as a consequence of negligence from the nurses' side. This example of her early research, which has been integrated into the monograph, shows her general approach to her topic. She creates new concepts to describe phenomena like general paralysis among mentally ill patients; her focus, however, is much more on the physical body, on medical and care taking practices concerning the physical body than what she promises in her introduction, namely, the investigation of the relationship between physical and mental illnesses $(1,2,10)$.

The book begins with a brief history of the body and mental disease, a term the author uses instead of "mental illness" to refer to the state of the physical illness in connection with the mind. Wallis's focus is concerned with mental illness only 
in the sense of a brain damage or some alterations in the structure (tissues) of the brain, but there is no reference to insanity or madness as a cultural concept in the main, analytical part of the text. It is only in her introduction to the topic that she mentions how madness is not solely examined in psychiatry but also in literature, and she refers to some scholars who did analyze the representations of madness in fiction. By including such aspects in the introduction, the author gives a misleading idea about the contents of the book. The reader, nevertheless, might direct their attention from the main theme of the book - which is the examination of the body in the nineteenth century, employing methods such as photography, the preservation of the brain and the documentation of records, related to the investigation of the living and the dissection of the dead body of mentally ill patients - to reflecting on mental illness from a psychological perspective. In this sense, the clinical psychiatric thread that the book seems to suggest and follow is but a pathological analysis of the diseased body. Hence, the mentally diseased patients' brain or all the other illnesses (syphilis, general paralysis, etc.) were not investigated in a way that one could get closer to the relationship of the mental issue and its traces in the body but other, not mental illnesses were examined instead. Mental illnesses that were supposed to be scrutinized by doctors in order to find the connection between mental illness and physical disease were not the core of the examination but rather bodily diseases were examined in patients who suffer from a mental illness.

Wallis argues that the medical practices of the nineteenth century can transform the body into a scientific object. At the beginning of the book, her argument suggests that the body is under-explored in the history of medicine but at the same time she contends that throughout history bodies have proven to be the focus point of researchers in the field of medicine and psychiatry (3). In fact, Wallis's opinion oscillates between the importance of the physical examination of patients' bodies (alive or dead) and the contribution of nineteenth-century scientific research to the discovery of the medical bond between the body and the mental status of patients.

Each chapter of the book tackles a different body part of the diseased patients and the medical practices that were executed on the bodies of mental patients to reach scientific discoveries. The first chapter, entitled "Skin," reveals the importance of photography as a source of information for doctors to get to know the internal workings of physical illnesses that reveal themselves on the skin, such as red spots on the body in the case of syphilis. After the exploration of the skin, the muscular system is addressed in the second chapter. Her focus is specifically on general paralysis both before and after death, on the difficulties in speaking and moving, and also on the socio-economic consequences of the loss of physical strength. In the following chapters, Wallis concentrates on the technological innovations that 
doctors incorporated in their practice to detect bone fractures before and after the patient's death, based on the assumption that the bones of general paralytic patients would be weaker than those of non-paralytic patients. She also emphasizes the importance of the brain in the Victorian asylum because it is considered the most important body part that allows researchers to see the impact of general paralysis on the brain of the patient and to conduct further postmortem research by resorting to brain preservation. In this regard, doctors had to figure out new scientific methods and invent new devices such as the microscope to investigate "the physical evidence" of mental disease in order to discover the causes of general paralysis. The last chapter, "Fluid" describes how fluids such as blood and urine were used and analyzed by researchers to understand the mechanisms of general paralysis.

Considering the brain, Wallis asserts that clinical and pathological methods were used by doctors to spot the softening of the brain as an indicator of mental disease. However, the author's exploration of the institutional practices is not thorough in the sense that she solely reports what was examined on patients in nineteenthcentury asylums, instead of stating the extent to which these practices are effective in finding the connection between mental disease and physical health. Moreover, she seems to be lost between providing a social history of psychiatry-which does not primarily focus on the body - and those experiences of diseased patients that are related to mental illness and physical disease.

Reading the title from the perspective of what is argued for in the introduction, the title might be slightly misleading because the preface explicitly states that the monograph will investigate the physical relationship between the body and the mental disease of patients, and so the title might be somewhat ambiguous as it can be both read as an investigation of the body without delving into the relation between the body and mental illness, and as a historical reading of mental problems in the Victorian period, especially as the phrase "mental health in historical perspective" precedes the main title on the cover page of the book. But because it is an examination of pathological and anatomical practices carried out on asylum patients' body parts, one might also form another assumption based on the introduction. In addition to that, syphilis and general paralysis are the most widely discussed diseases in the book, however, the title does not include general paralysis, which is after all the main topic and the most highlighted illness discussed throughout the investigation. The same thing applies to West Riding Asylum as the main institution chosen for the study, where the examinations of patients took place. Scholars interested in mental illnesses from biopolitical, cultural or socio-cultural perspectives may be disappointed since the book delves into the examination of the body in its medical and physical aspect more emphatically 
than into the field of mental illness as a psychological or psychosocial disease. Approaching the topic from both psychosocial and medical perspectives would diversify the type of readership and attract researchers in the field of humanities and social sciences as well as widen the horizons in the sense of moving from the patient's body and diseases to its effects on human psychology and society. Besides this, the book has a conclusion which seems to be a summary of previous chapters with the author's comments. In this way, the conclusion is as limited in scope as the monograph: it does not inspire new directions, it does not offer potential links to other areas of Victorian studies or the medical humanities, nor does it invite, or get engaged in, a dialogue with other fields.

While the writer answers a lot of questions, the exploration of bodily diseases in asylum patients shows some gaps; for instance, she fails to consider the gender perspective in her investigation given the fact that men's and women's physiology is different. And whereas the monograph has undeniable merits that deserve acknowledgement, namely that Wallis provides a historically informative account of the "neglected aspect of asylum history: the clinical and pathological investigation of the body" (16), the reader may miss the analysis of the institution's practices, or the outcome of doctors' investigations in finding physical proofs of mental illness that the author rhetorically promises and proposes to do from the beginning as the subject of inquiry.

Nevertheless, Wallis's refined style and careful choice of words make her writing comprehensive and subtle. She provides examples of real cases of patients in every chapter with illustrations and photos to explore the various practices carried out on patients with different diseases. Through the investigation of the records, practices, methods and concepts of the contemporary medical practice, Wallis not only focuses on asylum patients but she also considers the other side of this duality, the asylum doctor. It is true that patients are the backbone of the scientific study and without their bodies there would be no discovery, but doctors are keepers of the professional tradition by making portraits archived in West Riding hospital. Doctors are also the maintainers of balance in the sense that the author explicitly argues that there was a co-operation between patients and doctors, by giving the example that doctors recorded patients' feelings and delusions in order to study general paralysis. Mentally ill patients could spell out to doctors their concerns and sufferings, which were sometimes traumatic, especially in the case of chronic diseases.

Overall, Wallis's book is a window into the world of Victorian asylums not only as a space for isolating mentally ill patients but also as scientific spaces where the discovery of physical diseases in relation to the brain is made and the medical interaction between doctors and patients in physical examination to establish 
relationships of care is constructed. It is a significant contribution to body studies and Victorian studies since with its scientific terminology it can please specialists in the field of anatomy and medical psychiatry as well as students of medical sciences. I can recommend it to researchers and scholars who are interested in the clinical and pathological practices of nineteenth-century asylums and in the investigation of the body, as well as to readers who enjoy reading (about) human anatomy. 